\title{
PERFIL MOTIVACIONAL TEMPRANO DE ESTUDIANTES CON DIFICULTADES DE ACCESO AL LÉXICO Y COMPRENSIÓN LECTORA EN EDUCACIÓN PRIMARIA
}

\author{
Jessica Mercader Ruiz \\ Universitat Jaume I \\ mercader@uji.es \\ Ana Badenes-Gasset Presentación \\ Universitat Jaume I \\ Laura Abellán Roselló \\ Universitat Jaume I \\ Rebeca Siegenthaler Hierro \\ Universitat Jaume I
}

Fecha de Recepción: 9 Abril 2019

Fecha de Admisión: 30 Abril 2019

\begin{abstract}
RESUMEN
El objetivo del presente estudio retrospectivo consistió en determinar el perfil motivacional temprano de distintos grupos de estudiantes categorizados en función de su nivel de rendimiento lector en acceso al léxico y comprensión en $2^{\circ}$ de Educación Primaria. La muestra inicial estuvo compuesta por 208 preescolares de 5 a 6 años y sus tutores, de los cuales 179 volvieron a ser evaluados dos años más tarde. En Educación Infantil, los maestros y maestras cumplimentaron la escala de estimación Preschool Learning Behaviors Scale (PLBS; McDermott, Green, Francis, y Stott, 2000) que evalúa distintas conductas relacionadas con la motivación hacia el aprendizaje (competencia-motivación, atención-persistencia y actitud hacia el aprendizaje). Dos años más tarde, se administró la Batería de Evaluación de los Procesos Lectores (PROLEC-R; Cuetos, Rodríguez, Ruano, y Arribas, 2007). Los participantes se categorizaron en 4 grupos en función de su rendimiento en tareas de acceso al léxico y comprensión del PROLEC-R (Cuetos et al., 2007): dificultades en acceso al léxico ( $D A L, n=27$ ); dificultades en comprensión lectora ( $D C L, n=69$ ); dificultades en ambos dominios $(D A L+D C L=32)$ y rendimiento medio $(R M, n=51)$. Los resultados revelaron diferencias significativas entre los grupos en todas las variables de motivación hacia el aprendizaje evaluadas en Educación Infantil, con un peor funcionamiento de los grupos con dificultades en comparación con los estudiantes con rendimiento medio. Se destaca una mayor afectación de los grupos DAL+DCL y DAL en comparación con los estudiantes con DCL en la capacidad para persistir ante las tareas (atención-persistencia). Se comentan las implicaciones de estos hallazgos para la investigación y la práctica psicoeducativa.
\end{abstract}




\section{PERFIL MOTIVACIONAL TEMPRANO DE ESTUDIANTES CON DIFICULTADES DE ACCESO AL LÉXICO Y COMPRENSIÓN LECTORA EN EDUCACIÓN PRIMARIA}

Palabras clave: estudio longitudinal; dificultades lectoras; motivación; competencia percibida; persistencia; actitud hacia el aprendizaje

\section{ABSTRACT}

Early motivational profile of students with difficulties of access to lexico and reading comprehension in primary education. The aim of this retrospective study was to determine the early motivational profile of different groups of students categorized according to their reading performance level in lexicon access and understanding in 2nd year of Primary Education. The initial sample consisted of 208 preschoolers from 5 to 6 years old and their tutors, of whom 179 were evaluated again two years later. In Early Childhood Education, teachers completed the scale of the Preschool Learning Behaviors Scale (PLBS, McDermott, Green, Francis, \& Stott, 2000) that evaluates different behaviors related to motivation towards learning (competence-motivation, attentionpersistence and attitude towards learning). Two years later, the Battery for the Evaluation of Reading Processes was administered (PROLEC-R, Cuetos, Rodríguez, Ruano, \& Arribas, 2007). Participants were categorized into 4 groups based on their performance in tasks of lexicon access and understanding of PROLEC-R (Cuetos et al., 2007): difficulties in lexicon accessing (DLA, $n=27$ ); difficulties in reading comprehension (DRC, $n=69)$; difficulties in both domains $(D L A+D R C=32)$ and average performance (AP, $n=51$ ). The results revealed significant differences between groups in all the variables of motivation towards learning evaluated in Early Childhood Education, with a worse functioning of the groups with difficulties in comparison with the students with average performance. A greater affectation of the DLA + DRC and DLA groups is highlighted in comparison with the students with MRC in the ability to persist before the tasks (attention-persistence). The implications of these findings for research and psychoeducational practice are discussed.

Keywords: Iongitudinal study; reading difficulties; motivation; perceived competence; persistence; attitude towards learning

\section{ANTECEDENTES DE LA TEMÁTICA A TRATAR}

Aprender a leer es un objetivo clave de las etapas educativas iniciales ya que la lectura es una herramienta fundamental para el aprendizaje. Muchos estudiantes presentan dificultades en esta área que pueden tener consecuencias adversas en todos los órdenes de la vida (Hulme y Snowling, 2013). A pesar de la importancia de este aprendizaje, los resultados que ha obtenido España en el último informe PISA (MECD, 2015), sitúa al alumnado español en lectura por debajo de la media. Concretamente este informe, aunque reporta ligeras mejoras desde el año 2000, sitúa a España en el puesto 22 de los países. Estudios con muestra española, muestran que las Dificultades Específicas de Aprendizaje (DEA) tienen la mayor prevalencia en el ámbito de la Educación Especial (González et al., 2010). Concretamente, en el caso de la lectura, las dificultades en procesos básicos de lectura como el acceso al léxico presentan una prevalencia que oscila entre el $3.2 \%$ y $5.9 \%$ (Jiménez, Guzmán, Rodríguez, y Artiles, 2009). En el caso de los procesos superiores de comprensión lectora las cifras son todavía superiores, con un porcentaje del $20.2 \%$ de la población estudiantil (García, Jiménez, González, y Jiménez-Suárez, 2013; Jiménez et al., 2015).

El rendimiento lector y las dificultades que de este se derivan se puede ver afectado por distintos factores. La mayoría de los trabajos se han centrado en la influencia de factores cognitivos, tales como las aptitudes y la inteligencia (Castejón, 1996; McDermott, Goldberg, Stanley, y Glutting, 2006) o las funciones ejecutivas, destacando la memoria de trabajo y la velocidad de procesamiento (Altemeier, Abbott, y Berninger, 2008; Butterfuss y Kendeou 2018). Sin embargo, son menos los estudios que se han centrado investigar acerca de la importancia de aspectos motivacionales como las atribuciones, el autoconcepto, las orientaciones a meta, las estrategias de aprendizaje, la com- 
petencia percibida 0 el esfuerzo (Cho, Lee y Toste, 2018; Choi, 2005; González-Pienda et al., 2003; Kusdemir y Bulut, 2018) para el desarrollo de las dificultades lectoras. En esta línea, McDermott, Mordell y Stoltzfus (2001) establecen que la motivación y la persistencia son los predictores del éxito académico más importantes.

En relación con los perfiles motivacionales de niños y niñas con y sin problemas en lectura, algunos trabajos han encontrado diferencias en aspectos cognitivo-motivacionales como el autoconcepto, la autoestima o el estilo atribucional (véase Soriano, 2017). En lo que se refiere a las dificultades en procesos básicos de acceso al léxico, diferentes estudios reportan que los estudiantes que padecen dicha condición presentan un estilo motivacional desadaptativo. Concretamente, perciben menos la lectura como una meta de desarrollo personal y presentan una aproximación superficial al aprendizaje que se caracteriza por una motivación extrínseca (Polychroni, Koukoura, y Anagnostou, 2006; Zisimopoulos y Galanaki, 2009). En lo que se refiere a etapas tempranas del desarrollo, un reciente estudio de McDermott, Rikoon, y Fantuzzo (2014) concluye que factores motivacionales como la competencia percibida y la persistencia, diferencia la trayectoria de niños con y sin dificultades en el rendimiento lector inicial desde la etapa de Educación Infantil hasta el segundo curso de Educación Primaria. En relación a la comprensión lectora, distintos trabajos apuntan que los estudiantes con dificultades en esta área tienen una menor motivación intrínseca en comparación a sus iguales sin problemas (Logan, Medford y Hughes, 2011; Sideridis, 2005),

En definitiva, algunos estudios han mostrado rasgos motivacionales específicos de estudiantes con distintas dificultades lectoras en Educación Primaria y Secundaria. No obstante, son necesarios más trabajos que analicen estas diferencias en variables de motivación inicial hacia el aprendizaje, desde una aproximación longitudinal que abarque la etapa de Educación Infantil y contemple las dificultades en los distintos procesos relacionados con la lectura.

\section{OBJETIVOS DE LA INVESTIGACIÓN}

El objetivo del presente estudio retrospectivo consistió en determinar el perfil motivacional en Educación Infantil (T1) de distintos grupos de estudiantes categorizados en función de su nivel de rendimiento lector en acceso al léxico y comprensión en 2ํ de Educación Primaria (T2).

\section{MUESTRA Y/O PARTICIPANTES}

La muestra inicial del estudio (T1) estuvo conformada por 208 estudiantes (52.4\% varones; $47.6 \%$ niñas) de 5 y 6 años [Media (meses) $=70.21$, DT $=3.56$ ] y sus maestros/as, seleccionados mediante un método de muestro aleatorio simple. Ninguno de los participantes presentaba discapacidad intelectual [Media ( $\mathrm{Cl}$ Equivalente $)=98.28$; DT $=12.34$; rango $=70-128$ ] ni deficiencias sensoriales graves, anomalías neurobiológicas, trastornos psicológicos o deprivación sociocultural. El $88 \%$ de los participantes poseía nacionalidad española. Todos los sujetos hablaban y comprendían el castellano, independientemente de su nacionalidad. Dos años más tarde (T2), se retornó a los centros y se conformó la muestra definitiva del estudio ( $n=179 ; 86.6 \%$ de la muestra inicial). Los participantes tenían edades comprendidas entre 7 y 8 años [Edad media (meses) $=94.16$; DT = 3.48]. El $19.1 \%$ de los participantes asistía a sesiones con especialistas en los respectivos centros escolares (63.5\% centros públicos; $36.5 \%$ centros concertados): Apoyo Educativo $(7.7 \%)$, Educación Compensatoria (1.9\%), Pedagogía Terapéutica (3.3\%), Audición y Lenguaje (3.8\%) y tratamiento combinado (2.4\%).

La muestra se dividió en función de las puntuaciones estandarizadas obtenidas en los índices principales de las tareas de lectura de palabras, lectura de pseudopalabras y comprensión de textos del PROLEC-R (Cuetos et al., 2007) en el T2: "N = lector normal"; " $D=$ con dificultad"; "DD = con dificultad severa". Partiendo de estas directrices, se formaron cuatro grupos: dificultades de acce- 

LECTORA EN EDUCACIÓN PRIMARIA

so al léxico (DAL), dificultades de comprensión lectora ( $D C L)$, dificultades en ambos dominios (DAL+DCL) y rendimiento medio (RM). El grupo con DAL estuvo conformado por los sujetos con una puntuación "D o DD" en los índices principales de las tareas de lectura de palabras, lectura de pseudopalabras 0 ambas, pero con un nivel adecuado de comprensión $(n=27)$. Los participantes cuyas puntuaciones fueron "D o DD" en el índice principal de comprensión de textos, pero sin dificultades en las tareas de acceso al léxico, formaron parte del grupo DCL $(n=69)$. El grupo $D A L+D C L$ estuvo compuesto por niños y niñas con puntuaciones "D o DD" en todas las tareas ( $n=32$ ). Aquellos sujetos que obtuvieron una puntuación "N" en los índices principales de las tres tareas conformaron el grupo de RM $(n=51)$. Se encontraron diferencias estadísticamente significativas entre los grupos en la variable $\mathrm{Cl}$ equivalente [Wilks' Lambda $(\Lambda)=.82, \mathrm{Fg}, 418=3.87, p<.001$, $\left.\eta^{2} p=.063\right]$ que fueron tenidas en cuenta en los posteriores análisis.

\section{METODOLOGÍA}

\section{Instrumentos}

T1: Motivación inicial hacia el aprendizaje.

Los/las maestros/as de Educación Infantil cumplimentaron el cuestionario de estimación comportamental Preeschool Learning Behaviour Scale (PLBS; McDermott et al., 2000) con el objetivo de evaluar las conductas tempranas de motivación hacia el aprendizaje. La escala se compone de por 29 ítems agrupados en 3 subescalas: competencia-motivación, que estima las conductas relacionadas con la anticipación del éxito (i.e. "Parece que se refugia en una actitud de impotencia"); atención-persistencia, que evalúa la capacidad de persistir realizando una tarea hasta llegar a completarla; (i.e. "Se implica en las tareas en la medida que se espera que lo hiciera para su edad"); y actitud hacia el aprendizaje, cuyos ítems se relacionan con la voluntad de participar en las actividades de aprendizaje, mostrando predisposición hacia los elementos que lo conforman (i.e. "Muestra poco interés en agradar al profesor"). Las respuestas se emiten mediante una escala tipo Likert ( 0 = "Muy a menudo", 1 = "A veces", 2 = "Casi nunca"). Se calculó el nivel de fiabilidad total del cuestionario para la presente muestra (Alfa de Cronbach $=.86$ ). Se utilizó la puntuación directa en cada una de las subescalas para los análisis.

T2: Rendimiento en acceso al léxico y comprensión lectora.

Con el objetivo de categorizar a los sujetos en función de su rendimiento en tareas de acceso al léxico y comprensión lectora al finalizar el primer ciclo de Educación Primaria, se seleccionaron las tareas de lectura de palabras, lectura de pseudopalabras y comprensión de textos de la batería POLEC-R (Cuetos et al., 2007). En las tareas de lectura de palabras y pseudopalabras, se pide al sujeto que lea en voz alta una serie de 40 elementos respectivamente, contabilizando los aciertos y el tiempo empleado. En la tarea de compresión de textos, el participante debe leer en voz alta cuatro textos (dos narrativos y dos expositivos) y al finalizar el examinador realiza 4 preguntas sobre cada uno de ellos (literales e inferenciales). La fiabilidad test-retest de la prueba se ha constatado en .79, además de contar con elevados índices de validez (Cuetos et al., 2007). Se utilizaron las puntuaciones estandarizadas en los índices principales de las tres tareas para la asignación de los sujetos a los grupos de la presente investigación.

\section{Procedimiento}

Previo a la administración de pruebas se obtuvo autorización por parte de la Consellería de Educación, Cultura y Deporte y el Comité Ético de la Universitat Jaume I, así como se contó con el permiso de los centros educativos y el consentimiento informado de padres/madres/tutores. En el T1, los/las maestros/as cumplimentaron la escala PLBS (McDermott et al., 2000). En el T2, se retor- 
nó a los centros escolares para la aplicación de la batería PROLEC-R (Cuetos et al., 2007) a los sujetos. La administración de la batería se llevó a cabo en horario escolar, en aulas cedidas por los centros educativos que reunían las condiciones idóneas para la evaluación psicopedagógica y sin interferir con actividades significativas del curriculum. Comprendió dos sesiones individuales de 25 minutos y fue realizada por parte de evaluadores familiarizados con el uso de la prueba.

\section{Análisis estadísticos}

Para la realización de los análisis estadísticos, se empleó el programa Statistical Package for the Social Science, versión 22.00 (SPSS Inc., Chicago Illinois, USA). Para comprobar las diferencias en las variables de motivación hacia el aprendizaje evaluadas en el T1 entre los distintos grupos generados en función del rendimiento lector en el T2 (DAL, DCL, DAL+DCL y RM), se llevó a cabo un análisis multivariado de la covarianza (MANCOVA), introduciendo el $\mathrm{Cl}$ equivalente como variable de control. Se comprobaron los efectos principales $(p<.05)$, se realizaron comparaciones post-hoc por el método Bonferroni y se calculó el valor de $\eta^{2} p$ para comprobar la fuerza de la asociación.

\section{RESULTADOS ALCANZADOS}

En la Tabla 1 se recogen los análisis referidos a las diferencias en las variables de motivación incial hacia el aprendizaje. El efecto principal de grupo resultó estadísticamente significativo [Wilks' Lambda $\left.(\Lambda)=.82, \mathrm{~F}_{9}, 418=3.87, p<.001, \eta^{2} p=.063\right]$. Los ANCOVA's de confirmación mostraron diferencias estadísticamente significativas en todas las subescalas: competencia-motivación $\left(\mathrm{F}_{3}\right.$, $\left.174=10.49, p<.001, \eta^{2} p=.153\right) ;$ atención-persistencia $\left(F_{3,174}=9.25, p<.001, \eta^{2} p=.138\right)$; Actitud hacia el aprendizaje $\left(\mathrm{F}_{3,174}=3.45 p=.018, \eta^{2} p=.056\right)$.

Las comparaciones por pares a posteriori (Bonferroni) revelaron diferencias estadísticamente significativas entre el grupo con RM y el grupo con DAL en todas las variables motivacionales analizadas (competencia-motivación, $p=.003$; atención-persistencia, $p=.001$; actitud hacia el aprendizaje, $p=.027)$. El grupo RM presentó también diferencias estadísticamente significativas con el grupo de $\mathrm{DAL}+\mathrm{DCL}$ en las subescalas de competencia-motivación $(p<.001)$ y atención-persistencia $(p<.001)$ y con el grupo DCL para la variable competencia-motivación $(p<.001)$. Se encontraron diferencias estadísticamente significativas entre los grupos DCL y DAL+DCL $(p<.001)$, y entre DCL y DAL $(p<.001)$ en atención-persistencia.

Tabla 1. Comparaciones entre los grupos con y sin dificultades lectoras en variables motivacionales

\begin{tabular}{|c|c|c|c|c|c|c|c|}
\hline & $\begin{array}{l}\mathrm{DAL} \\
\mathrm{n}=27\end{array}$ & $\begin{array}{l}\text { DCL } \\
n=69\end{array}$ & $\begin{array}{c}\mathrm{DAL}+\mathrm{DCL} \\
\mathbf{n}=32\end{array}$ & $\begin{array}{c}\mathrm{RM} \\
\mathbf{n}=51\end{array}$ & & & \multirow{2}{*}{$\begin{array}{c}\text { Diferencias entre } \\
\text { grupos }\end{array}$} \\
\hline & $\begin{array}{c}\mathrm{M} \\
(\mathrm{DT})\end{array}$ & $\begin{array}{c}\mathrm{M} \\
(\mathrm{DT})\end{array}$ & $\begin{array}{c}\mathrm{M} \\
(\mathrm{DT})\end{array}$ & $\begin{array}{c}\mathrm{M} \\
(\mathrm{DT})\end{array}$ & $F_{3,174}$ & $\eta^{2} p$ & \\
\hline $\begin{array}{l}\text { Competencia- } \\
\text { Motivación }\end{array}$ & $\begin{array}{l}17.41 \\
(4.93)\end{array}$ & $\begin{array}{l}18.67 \\
(4.04)\end{array}$ & $\begin{array}{l}15.22 \\
(5.25)\end{array}$ & $\begin{array}{l}20.35 \\
(1.98)\end{array}$ & $10.49 * *$ & .153 & $\begin{array}{c}\mathrm{RM}>\mathrm{DAL} \\
\mathrm{RM}>\mathrm{DCL} \\
\mathrm{RM}> \\
\mathrm{DAL}+\mathrm{DCL}\end{array}$ \\
\hline $\begin{array}{l}\text { Atención- } \\
\text { Persistencia }\end{array}$ & $\begin{array}{l}12.22 \\
(4.15)\end{array}$ & $\begin{array}{l}13.70 \\
(4.19)\end{array}$ & $\begin{array}{l}11.00 \\
(4.50)\end{array}$ & $\begin{array}{l}15.49 \\
(2.67)\end{array}$ & $9.25 * *$ & .138 & $\begin{array}{c}\mathrm{RM}>\mathrm{DAL} \\
\mathrm{RM}> \\
\mathrm{DAL}+\mathrm{DCL} \\
\mathrm{DCL}>\mathrm{DAL} \\
\mathrm{DCL}> \\
\mathrm{DAL}+\mathrm{DCL}\end{array}$ \\
\hline $\begin{array}{l}\text { Actitud hacia } \\
\text { el aprendizaje }\end{array}$ & $\begin{array}{l}11.44 \\
(2.51)\end{array}$ & $\begin{array}{l}12.20 \\
(2.34)\end{array}$ & $\begin{array}{l}11.50 \\
(2.25)\end{array}$ & $\begin{array}{l}12.88 \\
(1.87)\end{array}$ & $3.45 *$ & .056 & $\mathrm{RM}>\mathrm{DAL}$ \\
\hline
\end{tabular}

Nota: $*=p<.05 ; * * p<.001$ 


\section{PERFIL MOTIVACIONAL TEMPRANO DE ESTUDIANTES CON DIFICULTADES DE ACCESO AL LÉXICO Y COMPRENSIÓN LECTORA EN EDUCACIÓN PRIMARIA}

\section{DISCUSIÓN}

Esta investigación se propuso como objetivo examinar las diferencias en las variables motivacionales evaluadas a los 5 años en niños con dificultades lectoras (de acceso al léxico, de comprensión lectora y ambas) y alumnos sin dificultades lectoras en $2^{0}$ de Educación Primaria. En la línea del trabajo realizado por McDermott et al. (2014), el grupo sin dificultades es el que ha obtenido mejores resultados en todas las variables de motivación evaluadas. Es decir, el grupo de rendimiento lector medio en 2ํㅜ de Educación Primaria presenta en Educación Infantil una mayor autocompentencia percibida, una actitud más positiva hacia el aprendizaje en general y eran más persistentes que los grupos con dificultades lectoras dos años después en las diferentes tareas escolares que se les planteaban. Sin embargo, mucho menores son las diferencias entre los tres grupos con dificultades.

Específicamente, la autocompetencia percibida es la variable motivacional que diferencia significativamente al grupo de rendimiento medio de los tres grupos con dificultades. Este resultado es acorde con el obtenido en el trabajo de Cho et al. (2018), en el que se destaca la importancia de la competencia percibida en la explicación del rendimiento lector de estudiantes con dificultades. La persistencia, por su parte, discrimina de forma significativa tanto al grupo con rendimiento medio como al grupo con dificultades de comprensión de los dos grupos con dificultades de acceso (solo y combinado con problemas de comprensión). Por último, en la actitud hacia el aprendizaje en Educación Infantil, únicamente aparecen diferencias significativas entre el grupo sin dificultades y el grupo con dificultades de acceso al léxico. El hecho de que los grupos con dificultades de acceso al léxico hayan obtenido valoraciones sobre su motivación en Educación Infantil más bajas que el resto de los grupos apuntaría a que la motivación afecta especialmente a los procesos de acceso al léxico en esta etapa educativa, en la línea de otros trabajos que han denotado la presencia de problemas motivacionales en niños con dificultades en procesos básicos en otras etapas educativas (Polychroni et al., 2006; Soriano, 2017; Zisimopoulos y Galanaki, 2009).

El presente trabajo resulta innovador por su diseño longitudinal con una muestra formada por niños de Educación Infantil. Como limitación cabe destacar que la herramienta utilizada para evaluar la motivación de alumnado es un cuestionario que se administró a los maestros. Sería interesante incorporar otros métodos de evaluación en los que la motivación fuera evaluada a través del propio niño 0 de otras personas cercanas. Futuras investigaciones, deberían analizar también las diferencias entre estos mismos grupos en etapas posteriores en las que las dificultades pueden estar más afianzadas, así como que incluyeran otras variables (intrapersonales e interpersonales) que han mostrado su influencia en el desarrollo de las dificultades lectoras (Altemeier et al., 2008; Butterfuss y Kendeou, 2018; Castejón, 1996; McDermott, et al., 2006).

Para algunos autores, la falta de motivación es la principal causa del fracaso en lectura (Lepola, Poskiparta, Laakkonen y Niemi, 2005). Sin duda son muchas las causas que llevan al fracaso en cada niño o niña. Pero también parece claro que, desde modelos preventivos, es fundamental que las intervenciones destinadas a terminar con el fracaso y las dificultades lectoras tengan en cuenta el papel de la motivación en el desarrollo de las mismas (Morgan et al., 2008). Desde este planteamiento es muy importante que tanto las familias como el personal docente tengan información adecuada sobre la importancia que tiene la motivación en la prevención de las futuras dificultades lectoras. Resulta relevante que, tanto en los hogares como en las aulas, se propicien ambientes de aprendizaje que fomenten el protagonismo de los niños, ya que, si estos lo perciben, estarán más motivados para continuar con esta labor, y aumentará su interés y voluntad para aprender cada vez de forma más autónoma. 


\section{CONCLUSIONES}

Se concluye que la motivación en Educación Infantil, tiene una influencia notable sobre el rendimiento lector posterior y sus dificultades. Los resultados revelaron un peor funcionamiento de los grupos con dificultades en $2^{0}$ de Educación Primaria en comparación con los estudiantes con rendimiento medio en todas las variables de motivación hacia el aprendizaje evaluadas en Educación Infantil. Se destaca una mayor afectación de los grupos con dificultades de acceso al léxico (con y sin dificultades de comprensión añadidas) en comparación con los estudiantes con problemas de comprensión en la capacidad para persistir ante las tareas.

\section{REFERENCIAS BIBLIOGRÁFICAS}

Altemeier, L. E., Abbott, R. D., y Berninger, V. W. (2008). Executive functions for reading and writing in typical literacy development and dyslexia. Journal of clinical and experimental neuropsychology, 30(5), 588-606.

Butterfuss, R., y Kendeou, P. (2018). The role of executive functions in reading comprehension. Educational Psychology Review, 30(3), 801-826.

Castejón, J. L. (1996). Determinantes del rendimiento académico de los estudiantes y de los centros educativos: modelos y factores. Alicante: Editorial Club Universitario.

Cho, E., Lee, M., y Toste, J. R. (2018). Does perceived competence serve as a protective mechanism against performance goals for struggling readers? Path analysis of contextual antecedents and reading outcomes. Learning and Individual Differences, 65, 135-147.

Choi, N. (2005). Self-efficacy and selfconcept as predictors of college students' academic performance. Psychology in the Schools, 42(2), 197-205.

Cuetos, F., Rodríguez, B., Ruano, E. y Arribas, D. (2007). PROLEC-R, Batería de Evaluación de los Procesos Lectores, Revisada. (4a Edición). Madrid: TEA Ediciones.

García, E., Jiménez, J. E., González, D. y Jiménez-Suárez, E. (2013). Problemas de comprensión en el alumnado de Educación Primaria y Educación Secundaria Obligatoria: un estudio de prevalencia en español. European Journal of Investigation in Health, Psychology and Education, 3(2), 113-123.

González-Pienda, J. A., Núñez, J. C., Álvarez, L., Roces, C., González-Pumariega, S., González, P., y Bernardo, A. (2003). Adaptabilidad y cohesión familiar, implicación parental en conductas autorregulatorias, autoconcepto del estudiante y rendimiento académico. Psicothema, 15(3), 471477.

Hulme, C., y Snowling, M. J. (2013). Learning to read: What we know and what we need to understand better. Child Development Perspectives, 7, 1-5.

Instituto de Evaluación (2015). Marcos y Pruebas de Evaluación de PISA. Volumen I: resultados en Matemáticas, Lectura y Ciencias. Madrid: Ministerio de Educación.

Jiménez, J. E., Crespo, P., Rodríguez, C., Díaz, A., García, E. y González, D. (2015). Prevalencia de las dificultades específicas de aprendizaje en la Educación Secundaria Obligatoria. European Journal of Education and Psychology, 3(2), 317-327.

Jiménez, J. E., Guzmán, R., Rodríguez, C., y Artiles, C. (2009). Prevalencia de las dificultades específicas de aprendizaje: la dislexia en español. Annals of Psychology, 25(1), 78-85.

Ku demir, Y., y Bulut, P. (2018). The Relationship between Elementary School Students' Reading Comprehension and Reading Motivation. Journal of Education and Training Studies, 6(12), 97110.

Lepola, J., Poskiparta, E., Laakkonen, E., y Niemi, P. (2005). Development of and relationship between phonological and motivational processes and naming speed in predicting Word recog- 
nition in grade 1. Scientific Studies of Reading, 9, 367-399.

Logan, S., Medford, E., y Hughes, N. (2011). The importance of intrinsic motivation for high and low ability readers' reading comprehension performance. Learning and Individual Differences, 21(1), 124-128.

McDermott, P. A., Goldberg, M. M., Watkins, M. W., Stanley, J. L. y Glutting, J. J. (2006). A Nationwide Epidemiologic Modeling Study of LD Risk, Protection, and Unintended Impact. Journal of Learning Disabilities, 39(3), 230-251.

McDermott, P. A., Green, L. F., Francis, J. M., y Stott, D. H. (2000). PLBS; Preschool Learning Behaviors Scale. Philadelphia: Edumetric \& Clinical Science.

McDermott, P. A., Mordell, M., y Stoltzfus, J. C. (2001). The organization of student performance in American schools: Discipline, motivation, verbal learning, nonverbal learning. Journal of Educational Psychology, 93(1), 65-76.

McDermott, P. A., Rikoon, S. H., y Fantuzzo, J. W. (2014). Tracing children's approaches to learning through Head Start, kindergarten, and first grade: Different pathways to different outcomes. Journal of Educational Psychology, 106(1), 200-213.

Polychroni, F., Koukoura, K., y Anagnostou, I. (2006). Academic self concept, reading attitudes and approaches to learning of children with dyslexia: do they differ from their peers?. European Journal of Special Needs Education, 21(4) 415-430.

Sideridis, G. D. (2005). Attitudes and motivation of poor and good spellers: Broadening planned behavior theory. Reading and Writing Quarterly, 21, 87-103.

Soriano, M. (2017). Dificultades en el aprendizaje. Granada: Editorial GEU.

Zisimopoulos, D. A., y Galanaki, E. P. (2009). Academic intrinsic motivation and perceived academic competence in Greek elementary students with and without learning disabilities. Learning Disabilities Research \& Practice, 24(1), 33-43. 